\title{
Blessures et intoxications associées à la consommation de méthamphétamine : surveillance sentinelle à l'aide de la plateforme électronique du Système canadien hospitalier d'information et de recherche en prévention des traumatismes (eSCHIRPT), 2011 à 2019
}

\author{
Steven R. McFaull, M. Sc.; André Champagne, M.S.P.; Wendy Thompson, M. Sc.; Felix Bang, M.S.P.
}

Diffuser cet article sur Twitter

\begin{abstract}
Résumé
Des données sur les consultations à un service des urgences pour des blessures et des intoxications liées à la méthamphétamine entre le $1^{\mathrm{er}}$ avril 2011 et le 9 août 2019 ont été saisies dans 19 sites sentinelles au Canada pour des patients de tous âges.

On a recensé au total 1093 cas (97,6/100000 cas de l'eSCHIRPT) (dont 59,4 \% de patients de sexe masculin). La proportion d'intoxications était plus élevée chez les femmes que chez les hommes (71 \% contre 57,4\%). Les blessures et les intoxications accidentelles étaient en cause dans 14,8 \% des consultations à un service des urgences. Les blessures auto-infligées (durant ou à la suite de la consommation de méthamphétamine) étaient à l'origine de 11,4\% des cas. Les circonstances des blessures et les intoxications associées à la méthamphétamine étaient variées : blessures auto-infligées, traumatismes cérébraux liés aux chutes, maladies mentales, activités criminelles et diverses autres situations. Ces facteurs devraient être pris en compte dans la préparation des stratégies de réduction des méfaits.
\end{abstract}

Mots-clés : méthamphétamine, intoxication, blessure, surveillance, eSCHIRPT

\section{Introduction}

La méthamphétamine est un stimulant très addictif du système nerveux central. Aussi appelée " meth », " blue », « ice », « crystal meth », « crank » et "peanut », entre autres, la méthamphétamine se présente sous forme de poudre cristalline blanche sans odeur, au goût amer, qui se dissout dans l'eau ou l'alcool. La méthamphétamine a été mise au point dans les années 1930 à partir de sa substance mère : l'amphétamine. Elle était initialement utilisée dans les décongestionnants nasaux et les inhalateurs bronchiques ${ }^{1,2,3}$.
Comme l'amphétamine, la méthamphétamine réduit l'appétit, augmente la vigilance et l'énergie et favorise l'euphorie, l'activité physique, la volubilité, la désinhibition et la confiance. La consommation chronique de méthamphétamine peut entraîner des symptômes de psychose comme des hallucinations, la paranoïa et l'anxiété ${ }^{4}$.

À doses équivalentes, la méthamphétamine atteint le cerveau en plus grande quantité que l'amphétamine, ce qui en fait un psychostimulant plus puissant ${ }^{5}$. Ses effets sur le système nerveux central sont aussi plus durables et plus nocifs ${ }^{6}$. La méthamphétamine peut aisément être fabriquée avec
Points saillants

- Les services des urgences participants ont traité 1093 cas de blessures et de lésions liées à la méthamphétamine.

- La proportion de cas concernant les filles était de 71,4\% chez les 10 à 14 ans et de $63,7 \%$ chez les 15 à 19 ans.

- Chez les patients de 20 ans et plus, 70,4 \% étaient des hommes.

- Des 689 cas où d'autres substances étaient en cause (63\%), 40,9 \% impliquaient deux substances ou plus. Dans les cas où une seule substance autre que la méthamphétamine était en cause, celle-ci était l'alcool (24,4\%), le cannabis $(10,7 \%)$, la cocaïne $(7,7 \%)$, l'héroïne $(5,4 \%)$, un opiacé $(3,6 \%)$ ou une autre substance $(7,3 \%)$.

- La proportion d'intoxications était plus élevée chez les femmes, tandis que les blessures multiples étaient plus fréquentes chez les hommes.

- Des blessures auto-infligées ont été commises dans $11,4 \%$ des cas.

des précurseurs faciles à obtenir ${ }^{4}$. En raison de ces caractéristiques, elle constitue une substance à fort potentiel de dépendance.

De nombreux méfaits physiques, physiologiques et psychologiques ont été associés

Rattachement des auteurs :

Agence de la santé publique du Canada, Ottawa (Ontario), Canada

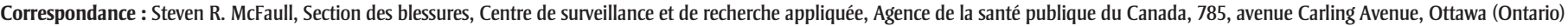
K1A 0K9; 613-404-1881; courriel : steven.mcfaull@canada.ca 
à la consommation et à la fabrication de méthamphétamine ${ }^{4,7,8,9-11}$. Ces méfaits varient selon le sexe $\mathrm{e}^{12}$.

Notre étude visait à décrire les circonstances à l'origine des consultations aux services des urgences pour des blessures et des intoxications associées à la consommation et à la fabrication de méthamphétamine.

\section{Méthodologie}

\section{Source de données}

La plateforme électronique du Système canadien hospitalier d'information et de recherche en prévention des traumatismes (eSCHIRPT) est un système de surveillance sentinelle des blessures et des intoxications traitées dans 11 hôpitaux pédiatriques et 8 hôpitaux généraux au Canada. L'eSCHIRPT, qui est administré par le Centre de surveillance et de recherche appliquée de l'Agence de la santé publique du Canada, saisit des renseignements détaillés dans des zones de texte, ce qui permet un codage très détaillé des circonstances et des mécanismes ${ }^{13}$. À l'inverse, les données administratives fondées sur la Classification statistique internationale des maladies et des problèmes de santé connexes, $10^{e}$ version, Canada $(\mathrm{CIM}-10-\mathrm{CA})^{14}$ ne sont pas suffisamment détaillées pour faire ressortir les cas liés à la méthamphétamine et ne fournissent pas d'information sur les circonstances des incidents.

\section{Extraction des cas}

Nous avons recherché, dans la base de données de l'eSCHIRPT, les cas (de tous âges) de blessures et d'intoxications liées à la méthamphétamine présents dans le système au 9 août 2019. Pour être retenus, ces cas devaient concerner un patient ayant consommé ou fabriqué de la méthamphétamine ou ayant été blessé par une personne soit sous l'influence de méthamphétamine soit fabriquant de la méthamphétamine (incluant les nourrissons nés d'une mère toxicomane et les enfants témoins). Comme ces personnes pouvaient avoir été blessées (par opposition à victimes d'intoxication), nous avons effectué une vaste recherche textuelle bilingue (en anglais et en français) pour recenser les cas à l'aide des champs d'information descriptive et d'identification de cette substance dans l'eSCHIRPT. Nous avons utilisé au départ une liste détaillée de 83 termes dans les deux langues officielles (disponible sur demande). Finalement, la majorité des cas $(82,1 \%)$ ont été recensés à partir de quatre mots-clés ou chaînes de mots : " meth ", " crystal meth », " speed » et " ice ». Pour épurer les données, nous avons exploré les champs d'information descriptive à l'aide d'expressions régulières du langage Perl (Practical Extraction and Report Language) ${ }^{15}$ dans le logiciel SAS 9.4 (SAS Institute Inc., Cary, Caroline du Nord, États-Unis). Toutes les expressions non pertinentes (p. ex. " speed bumps », "speeding ») ont été exclues.

L'ensemble de données épurées a été examiné manuellement et codé avec d'autres détails sur les circonstances (intervention policière, consommation d'autres substances, blessure auto-infligée, " en brosse », blessure accidentelle, etc.).

Nous avons réalisé les analyses statistiques à l'aide des logiciels SAS 9.4, Epi Info 7.2.0.1 (CDC, Atlanta, Géorgie, États-Unis) et Microsoft Excel (2013).

\section{Résultats}

\section{Aperçu}

Au total, 1093 patients $(97,6 / 100000$ cas de l'eSCHIRPT) ont été recensés pour la période du 1er avril 2011 au 9 août 2019, dont 59,4\% ( $\mathrm{n}=649)$ de sexe masculin. Les filles comptaient pour 71,4\% des cas chez les 10 à 14 ans et pour 63,7\% des cas chez les 15 à 19 ans. Chez les 20 ans et plus, ce sont les hommes qui prédominaient, avec 70,4\% des cas (voir la figure 1 pour la répartition normalisée selon l'âge et le sexe).

\section{Lieu}

Le lieu où la blessure était survenue a été indiqué dans 420 cas $(38,4 \%)$. Parmi ces lieux, 30,2 \% étaient le domicile du patient et $16,9 \%$ un autre domicile privé. Environ le quart $(26,4 \%)$ des incidents s'étaient produits à l'extérieur, sur une route, dans une rue ou dans un parc public. La blessure était survenue dans un domicile collectif dans 7,1\% des cas. Les autres incidents (environ $20 \%$ ) s'étaient produits dans des hôpitaux, des bars, des hôtels et divers autres endroits, chacun correspondant à seulement quelques points de pourcentage au total.

\section{Circonstances}

Constituant 14,8 \% des cas, les blessures accidentelles (excluant les effets toxiques des substances) étaient la circonstance la

\section{FIGURE 1}

Répartition normalisée ${ }^{\mathrm{a}}$ selon l'âgé et le sexe des consultations à un service des urgences associées à la consommation de méthamphétamine, eSCHIRPT, 2011-2019 $(n=1$ 093)

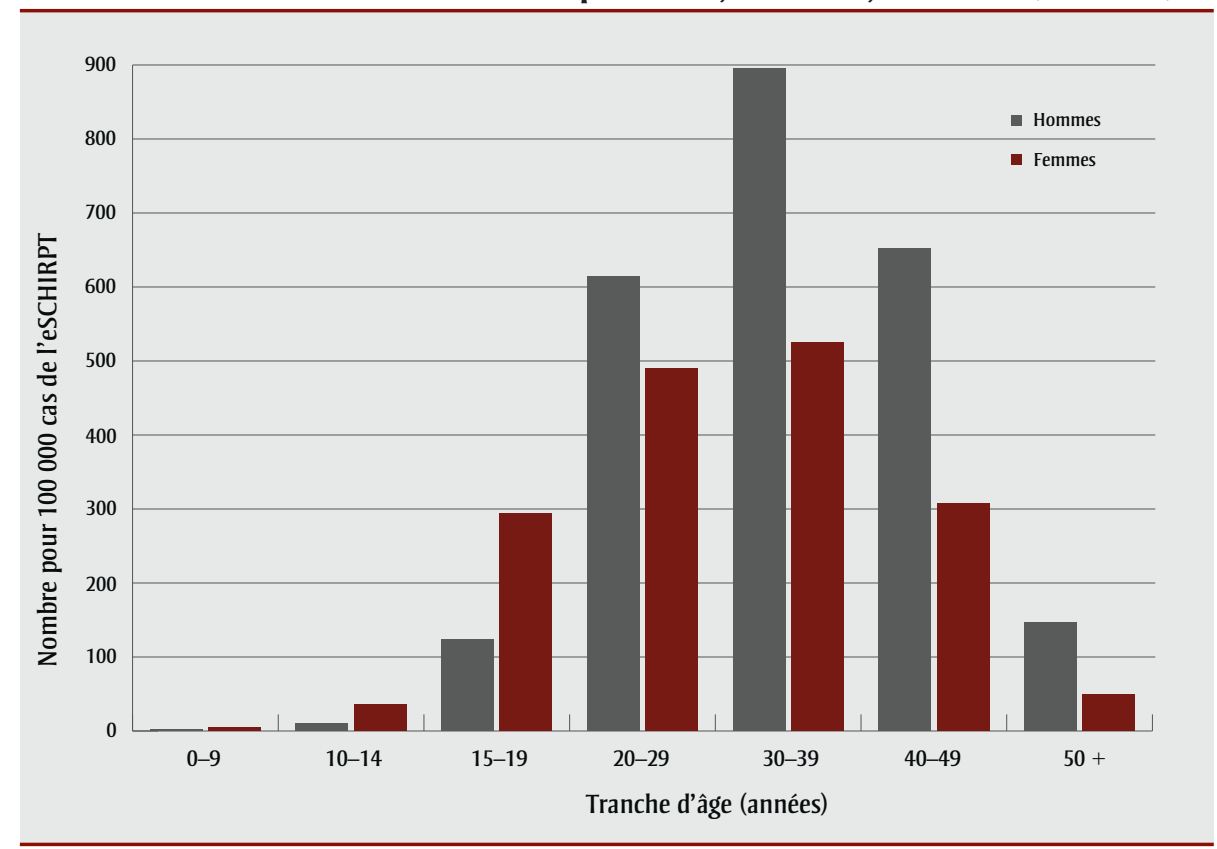

Abréviation : eSCHIRPT, plateforme électronique du Système canadien hospitalier d'information et de recherche en prévention des traumatismes.

${ }^{a}$ Exprimée en tant que proportion de tous les cas de l'eSCHIRPT dans une tranche d'âge donnée (× 100000$)$. 
plus fréquente à l'origine des consultations à un service des urgences (tableau 1). Parmi elles, 30,2 \% étaient des chutes. Des blessures auto-infligées, incluant les tentatives de suicide, avaient été commises dans $11,4 \%$ des cas. La plus grande proportion de blessures autoinfligées a été observée chez les 15 à 29 ans (données non présentées). Parmi les cas de blessure auto-infligée chez les 10 à 19 ans, $75 \%$ concernaient les filles; chez les 20 à 49 ans, 66,7\% concernaient les hommes. Sur les 1093 patients recensés, 10,8 \% avaient été impliqués dans une intervention policière ou une activité criminelle et 9,4\%, dans un acte de violence (familiale ou autre).

TABLEAU 1

Circonstances à l'origine des consultations à un service des urgences associées à la consommation de méthamphétamine, eSCHIRPT, 2011 à 2019 ( $n=1093)$

\begin{tabular}{|c|c|c|}
\hline \multirow[b]{2}{*}{$\begin{array}{l}\begin{array}{l}\text { Circonstances à l'origine des consultations à un service des } \\
\text { urgences }\end{array} \\
\end{array}$} & \multicolumn{2}{|c|}{ Nombre } \\
\hline & Fréquence (n) & Pourcentage (\%) \\
\hline Blessure accidentelle & 162 & 14,8 \\
\hline Chute & 49 & 4,5 \\
\hline Accident de la route & 32 & 2,9 \\
\hline Cycliste, piéton & 22 & 2,0 \\
\hline Autre blessure accidentelle ${ }^{\mathrm{b}}$ & 59 & 5,4 \\
\hline Blessure auto-infligée, suicide & 125 & 11,4 \\
\hline Intervention policière, activité criminelle ${ }^{c}$ & 118 & 10,8 \\
\hline Toxicomane connu ${ }^{\mathrm{d}}$ & 108 & 9,9 \\
\hline Consommation occasionnelle excessive d'alcool (« brosse ») & 106 & 9,7 \\
\hline Violence & 103 & 9,4 \\
\hline Violence familiale & 26 & 2,4 \\
\hline Autre forme de violence ${ }^{f}$ & 77 & 7,0 \\
\hline Patient retrouvé dans un état convulsif ou inconscient & 82 & 7,5 \\
\hline Patient retrouvé errant, sans abri & 62 & 5,7 \\
\hline Dépression, anxiété, maladie mentale & 59 & 5,4 \\
\hline Fête (dont rave) & 37 & 3,4 \\
\hline Demande d'aide & 9 & 0,8 \\
\hline Injection par une autre personne ou pilule donnée à son insug & 8 & 0,7 \\
\hline En cours de sevrage de méthamphétamine ${ }^{h}$ & 7 & 0,6 \\
\hline Lot de mauvaise qualité ou différent de la norme & 6 & 0,5 \\
\hline Consommation de méthamphétamine, SPP & 93 & 8,5 \\
\hline Autre $^{\mathrm{i}}$ & 8 & 0,7 \\
\hline Total & 1093 & 100,0 \\
\hline
\end{tabular}

Abréviations : eSCHIRPT, plateforme électronique du Système canadien hospitalier d'information et de recherche en prévention des traumatismes; SPP, sans plus de précisions.

a Chaque cas a été codé d'après l'information descriptive sur le patient de manière à correspondre à une seule catégorie.

${ }^{\mathrm{b}}$ Englobe les brûlures, les accidents de travail, les heurts et les coups, les intoxications et les piqûres d'aiguille.

' Englobe les poursuites policières, les introductions par effraction, le tapage et les personnes qui se jettent devant les voitures.

d Patient connu du personnel du service des urgences.

e Sur une période de plusieurs jours; englobe les piqueries et les repaires.

${ }^{\mathrm{f}}$ Attaques, bagarres et agressions sexuelles.

8 Personne s'étant fait injecter ou ayant ingéré de la méthamphétamine à son insu.

${ }^{\text {h }}$ Englobe les nourrissons nés d'une mère toxicomane.

' Englobe les lésions dues à un feu (lié à la consommation) ou survenues durant la fabrication ou la production de la substance; les femmes enceintes; les personnes atteintes de douleurs chroniques; les personnes ayant pris la substance pour s'aider à étudier; et les centres d'injection sécuritaire.

\section{Consommation d'autres substances}

Des 1093 patients recensés, 404 (37\%) avaient consommé uniquement de la méthamphétamine. Des 689 cas restants, $40,9 \%$ avaient consommé plusieurs autres substances (c.-à-d. au moins deux substances en plus de la méthamphétamine). Dans les cas où une seule autre substance était en cause, la distribution suivante a été établie : alcool $(24,4 \%)$, cannabis $(10,7 \%)$, cocaïne $(7,7 \%)$, héroïne $(5,4 \%)$, opiacé $(3,6 \%)$ et autre substance (p. ex. LSD, ecstasy, médicament psychoactif) $(7,3 \%)$.

\section{Blessures et intoxications - différences entre les sexes}

Les 1093 patients totalisaient 1389 blessures et intoxications, car 27,1\% d'entre eux présentaient plusieurs blessures. Les différences suivantes ont été constatées entre les sexes sur le plan des modes de blessure et d'intoxication : les patients de sexe masculin étaient plus nombreux que les patientes de sexe féminin à présenter plusieurs blessures $(32,8 \%$ contre $18,7 \%$; $p<0,001)$; en revanche, la proportion d'intoxications était plus élevée chez les patientes de sexe féminin que chez les patients de sexe masculin (71\% contre $57,4 \% ; p<0,001)$. De plus, les fractures étaient plus fréquentes chez les patients de sexe masculin que chez les patientes de sexe féminin $(21,5 \%$ contre $11,1 \%$; $p<0,005)$, tandis que les patientes de sexe féminin étaient plus nombreuses que les patients de sexe masculin à présenter des ecchymoses et des éraflures (16,3\% contre $7,9 \% ; p<0,005)$. Après l'évaluation au service des urgences, les patientes de sexe féminin étaient plus nombreuses que les patients de sexe masculin à recevoir le diagnostic « aucune blessure détectée » $(11,8 \%$ contre $4,4 \% ; p<0,005)$. La fréquence des traumatismes cérébraux était plus élevée chez les patients de sexe masculin que chez les patientes de sexe féminin $(9,8 \%$ contre $5,2 \%)$, mais en raison du faible nombre de cas, cette observation n'est pas statistiquement significative $(p=0,06)$. Treize patients avaient des engelures en raison d'une exposition prolongée au froid ("retrouvé inconscient à l'extérieur »). Onze de ceux-ci étaient de sexe masculin, mais cette différence entre les sexes n'est pas non plus statistiquement significative ( $p=0,21$ ), étant donné le faible nombre de cas. 


\section{Traitement au service des urgences}

Dans l'ensemble, 17,7 \% des patients de sexe masculin et $10,8 \%$ des patients de sexe féminin $(p<0,001)$ qui se sont présentés à un service des urgences ont été hospitalisés. Une proportion additionnelle de 2,9 \% des patients a été hospitalisée à la suite de circonstances autres qu'une blessure (p. ex. traitement de la toxicomanie).

\section{Analyse}

Les méfaits physiques et psychologiques de la méthamphétamine sont considérables tant pour les utilisateurs que pour leur famille et la société, c'est pourquoi cette substance pose un important problème de santé publique ${ }^{7,16}$.

La crise des opioïdes au Canada a révélé que plusieurs facteurs contribuent à la hausse rapide de la consommation de substances dans les populations ${ }^{17}$. Il faut donc assurer une surveillance permanente pour détecter les changements et mettre en application des stratégies de réduction des méfaits.

Les champs d'information descriptive de l'eSCHIRPT fournissent des renseignements contextuels et permettent de récolter des détails sur les circonstances des incidents (p. ex. intervention policière ou activité criminelle, violence familiale, état psychologique, blessure auto-infligée, itinérance, autre situation). Ces renseignements sont utilisables pour alimenter les mesures de promotion de la santé et de réduction des blessures. La base de données de l'eSCHIRPT a d'ailleurs déjà été utilisée pour décrire des cas d'intoxication suspectés d'être liés aux opioïdes ${ }^{18}$.

Nous avons constaté des différences entre les sexes sur le plan des intoxications, des blessures en général et des blessures autoinfligées. Ces différences (qui ont déjà été décrites ailleurs ${ }^{12}$ ) devraient être prises en compte dans la préparation d'initiatives de prévention.

\section{Points forts et limites}

L'eSCHIRPT ne saisit pas tous les incidents qui surviennent au Canada, mais ceux concernant les patients qui se présentent à un service des urgences participant. Comme la plupart des établissements participant à l'eSCHIRPT sont des hôpitaux pédiatriques (et qu'ils sont situés dans de grandes villes), certaines populations sont sous-représentées, par exemple les habitants des régions rurales (ce qui inclut certains peuples autochtones), les adolescents plus âgés et les adultes.

L'eSCHIRPT tient compte des personnes dont le décès est constaté à leur arrivée, mais non des personnes retrouvées mortes sur place et de celles décédées plus tard à l'hôpital. Il exclut aussi certains patients, ceux qui ne passent pas par le comptoir des admissions du service des urgences parce qu'ils doivent recevoir un traitement immédiat et ceux qui ne remplissent pas de formulaire de déclaration des blessures et des intoxications.

Enfin, dans les cas où plusieurs substances sont en cause, il n'est pas possible de déterminer les effets de chaque substance sur l'état du patient. Les différences entre les sexes sur les plans des comportements en matière de consommation de substances et des réponses aux substances pourraient s'expliquer par un biais lié aux individus s'étant rendus à un service des urgences $^{12,19}$.

\section{Conclusion}

Les différences sur le plan des circonstances, du sexe et de l'âge dans les cas de blessures et d'intoxications associées à la consommation et à la fabrication de méthamphétamine devraient être prises en compte dans la préparation des stratégies de réduction des méfaits.

\section{Remerciements}

Les auteurs tiennent à remercier James Cheesman pour l'extraction des données et Jaskiran Kaur et Aimée Campeau pour leur relecture du manuscrit.

\section{Conflits d'intérêts}

Les auteurs déclarent n'avoir aucun conflit d'intérêts.

\section{Contributions des auteurs et avis}

SRM : conceptualisation, analyse, méthodologie, rédaction du manuscrit initial; $\mathrm{AC}, \mathrm{WT}$ et $\mathrm{FB}$ : rédaction, révision et relecture.

Le contenu de l'article et les points de vue qui y sont exprimés n'engagent que les auteurs et ne correspondent pas nécessairement à ceux du gouvernement du Canada.

\section{Références}

1. Diversion Control Division. National Forensic Laboratory Information System (NFLIS): 2015 Annual Report. Springfield (VA): U.S. Department of Justice Drug Enforcement Agency; 2016.

2. Chomchai C, Chomchai S. Global patterns of methamphetamine use. Curr Opin Psychiatry. 2015;28(4):269-274. doi:10.1097/YCO.0000000000000168.

3. Levmetamfetamine [Internet]. Drugbank; 2015 [consulté le 3 octobre 2019]. En ligne à : https://www.drugbank.ca /drugs/DB09571

4. Sheridan J, Bennett S, Coggan C, Wheeler A, McMillan K. Injury associated with methamphetamine use: a review of the literature. Harm Reduct J. 2006;3:14. doi:10.1186/1477-7517 -3-14.

5. Panenka WJ, Procyshyn RM, Lecomte $\mathrm{T}$, et al. Methamphetamine use: a comprehensive review of molecular, preclinical and clinical findings. Drug Alcohol Depend. 2013;129(3):167-179. doi:10.1016/j.drugalcdep.2012.11.016.

6. Moszczynska A. Neurobiology and clinical manifestations of methamphetamine neurotoxicity. Psychiatr Times. 2016;33(9):16-18.

7. Darke S, Kaye S, McKetin R, Duflou J. Major physical and psychological harms of methamphetamine use. Drug Alcohol Rev. 2008;27:253-262. doi:10.1080/09595230801923702.

8. Charukamnoetkanok P, Wagoner MD. Facial and ocular injuries associated with methamphetamine production accidents. Am J Opthalmol. 2004; 138(5):875-876. doi:10.1016/j.ajo.2004 .05 .056 .

9. Lee JH, Farley CL, Brodrick CD, Blomquist PH. Anhydrous ammonia eye injuries associated with illicit methamphetamine production. Ann Emerg Med. 2003;41(1):157. doi:10.1067 /mem.2003.33. 
10. Davidson SB, Blostein PA, Walsh J, Maltz SB, Elian AE, Vandenberg SL. Resurgence of methamphetamine related burns and injuries: a followup study. Burns. 2013;39:119-125. doi:10.1016/j.burns.2012.03.003.

11. Swanson SM, Sise CB, Sise MJ, Sack DI, Holbrook TL, Paci GM. The scourge of methamphetamine: impact on a level I trauma center. J Trauma. 2007;63(3):531-537. doi:10.1097/TA $.0 \mathrm{~b} 013 \mathrm{e} 318074 \mathrm{~d} 3 \mathrm{ac}$.

12. Brecht ML, O’Brien A, von Mayrhauser C, Anglin MD. Methamphetamine use behaviors and gender differences. Addict Behav. 2004;29:89-106. doi: 10.1016/S0306-4603(03)00082-0.

13. Crain J, McFaull S, Thompson W, et al. Rapport d'étape. Le Système canadien hospitalier d'information et de recherche en prévention des traumatismes : un système de surveillance des blessures dynamique et novateur. Promotion de la santé et prévention des maladies chroniques au Canada 2016;36(6):127-133. doi:10.24095/hpcdp 36.6.02f.

14. Institut canadien d'information sur la santé. Classification statistique internationale des maladies et des problèmes de santé connexes, 10e version, Canada. Volume deux - Index alphabétique [Internet]. Ottawa (Ontario) : ICIS; 2015. En ligne à : https://www .cihi.ca/sites/default/files/icd_volume _two_2015_fr_0.pdf

15. Zhang Y. PERL regular expression in SAS macro programming. SAS Global Forum. 2011;159-2011:1-7.

16. McKetin R, Degenhardt L, Shanahan M, Baker AL, Lee NK, Lubman DI. Health service utilisation attributable to methamphetamine use in Australia: patterns, predictors and national impact. Drug Alcohol Rev. 2018;37:196-204. doi:10.1111/dar.12518.

17. Belzak L, Halverson J. La crise des opioïdes au Canada : une perspective nationale. Promotion de la santé et prévention des maladies chroniques au Canada 2018; 38(6):255-266. doi: 10.24095/hpcdp.38.6.02.
18. Do MT, Chang VC, Tibebu S, Thompson W, Ugnat AM. Surveillance des cas suspectés d'intoxication et de blessures liées aux opioïdes : tendances et contexte tirés du Système canadien hospitalier d'information et de recherche en prévention des traumatismes, mars 2011 à juin 2017. Promotion de la santé et prévention des maladies chroniques au Canada 2018;38(9):359-370. doi:10.24095/hpcdp .38.9.03f.

19. Mayo LM, Paul E, DeArcangelis J, Van Hedger K, de Wit H. Gender differences in the behavioral and subjective effects of methamphetamine in healthy humans. Psychopharmacology. 2019;236:2413-2423. 